\title{
Acute barium intoxication following ingestion of ceramic glaze
}

\author{
Merlin Thomas, David Bowie, Robert Walker
}

\begin{abstract}
Summary
A case of deliberate overdose of barium sulphide in a psychiatric setting is presented, with resulting flaccid paralysis, malignant arrhythmia, respiratory arrest and severe hypokalaemia, but ultimately with complete recovery. The degree of paralysis appears to be related directly to serum barium levels. The value of early haemodialysis, particularly with respiratory paralysis and hypokalaemia, is emphasised.
\end{abstract}

Keywords: barium; haemodialysis; poisoning

A 32-year-old ceramic artist was transferred from a psychiatric institute with a sudden onset of severe cramping abdominal pain and intractable vomiting and diarrhoea. She was known to have a bipolar affective disorder together with anorexia and was extremely emaciated. In addition, she had recently attempted suicide by stabbing herself with a potter's knife. It became apparent that 4 hours prior to admission she had ingested a potter's glaze containing $62 \%$ barium sulphide. On examination, she was incoherent, with excessive salivation and sweating. Her systolic blood pressure was $90 \mathrm{mmHg}$. Brief facial twitching was also noted. Laboratory results on admission revealed a severe hypokalaemia at $1.1 \mathrm{mmol} / 1$ with a mixed metabolic and respiratory acidosis $(\mathrm{pH} 7.1$, $\left.\mathrm{CO}_{2} 60 \mathrm{mmHg}, \mathrm{HCO}_{3} 17 \mathrm{mmol} / \mathrm{l}\right)$. A plain $\mathrm{X}$-ray of the abdomen showed extensive small bowel opacification. She received a gastric lavage together with oral magnesium sulphate as a laxative and binding agent. Over the next 3 hours she showed generalised weakness progressing to a flaccid paralysis and respiratory failure requiring ventilation. She developed non-sustained ventricular tachycardia requiring a brief period of cardiopulmonary resuscitation before spontaneous cardioversion. Saline diuresis and intravenous potassium supplementation was instituted without improvement of her weakness. On day three of her admission, she was commenced on haemodialysis using a dialysate potassium concentration of $4 \mathrm{mmol} / \mathrm{l}$. During the 4 hours of her dialysis there was dramatic reduction of her paralysis coincident with the reduction of her barium levels and she was able to be extubated (figure). She subsequently made a complete recovery with no residual neurological deficit.

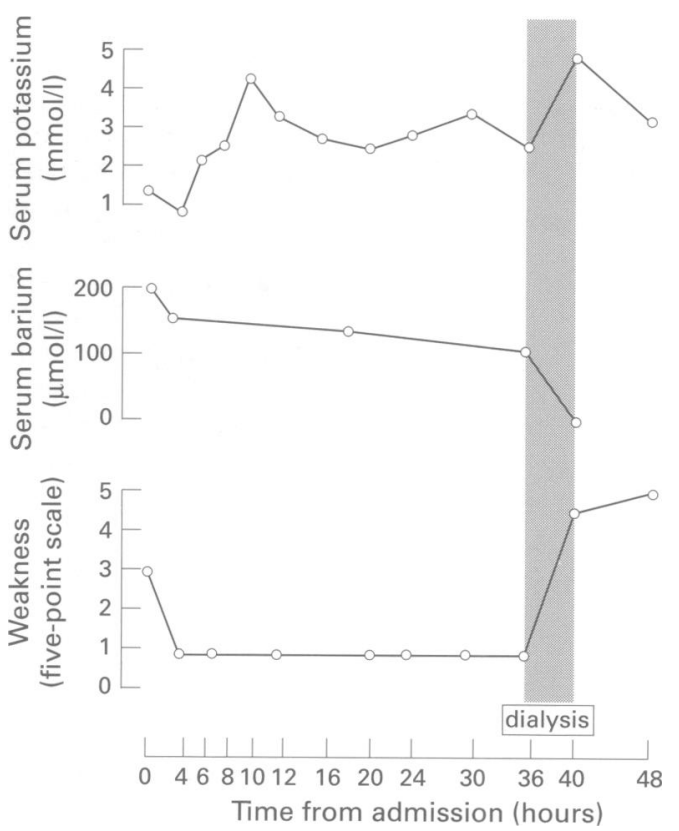

Figure Relation between serum barium, serum potassium and degree of strength following barium sulphide ingestion and haemodialysis

\section{Discussion}

Ceramic glazes are used to colour or finish clay pieces and are a mixture of silica, fluxes and colourants. Common fluxes used to lower the melting point of silica include lead, lithium, calcium and barium. Several cases record the accidental or deliberate ingestion of ceramic glazes by psychiatric patients during forms of recreational therapy involving the production of pottery. Some series have described up to $45 \%$ of such poisonings as having occurred in extended-care facilities among patients with impaired mental status. ${ }^{1}$ This has resulted in a call to ban the use of toxic glazes from all psychiatric and geriatric facilities. Product labelling in the USA specifically cautions against the use of toxic materials in such situations, but these safeguards are neither universally acknowledged nor observed. In the case described, a psychiatric patient with a professional background in ceramic ware was supplied with a familiar toxic substance in an unsupervised setting, despite the fact she was known to be suicidal. Her near-fatal ingestion is an important lesson in the provision of hazardous substances to mentally impaired or suicidal persons and the need for adequate 
staffing levels to ensure appropriate supervision for psychiatric patients.

Barium is well known to physicians as the largely innocuous and inert contrast agent barium sulphate. However, ingestion of ionic barium may result in severe toxicity and death. Initial symptoms of excessive salivation, severe abdominal pain, intractable vomiting and diarrhoea are manifest within 2-3 hours of ingestion. Progressive toxicity includes muscle twitching, peri-orbital and extremity paraesthesia, life-threatening arrhythmias and weakness progressing to convulsions and a flaccid paralysis. Profound hypokalaemia due to increased intracellular uptake may also be induced. ${ }^{2}$ It was initially thought that the marked reduction of potassium levels was the pathogenic factor in barium-induced weakness in the manner of hypokalaemic paralysis. In our case there was no correlation between serum potassium levels and degree of weakness (figure 1). Evidence now points to ionic barium itself inducing neuromuscular blockade and membrane depolarisation. ${ }^{3}$ The reduction in barium levels following haemodialysis coincident with the dramatic improvement in paraly-

1 Anon. Amendment to the Federal Hazardous Substances Act (Public Law 100-695). MMWR 1992;41(42):October 23.

2 Schorn TF, Olbright C, Sculer A, et al. Barium carbonate intoxication. Intens Care Med 1991;17:60-2. sis in this case supports a direct effect of barium.

Management of barium intoxication initially involves emesis and lavage. Activated charcoal does not bind barium and should not be used unless other agents are ingested or suspected. Oral magnesium sulphate may be useful to convert ionic barium in the gut into an insoluble sulphate form as well as to promote catharsis. Saline diuresis has also been recommended for barium poisoning. ${ }^{4}$ The correction of life-threatening hypokalaemia has been central to the management of barium intoxication. The potassium requirements are often enormous, with up to $420 \mathrm{mmol}$ per day needed to maintain serum levels. ${ }^{4}$ Haemodialysis shortens the serum half-life of barium restoring muscle strength and obviating prolonged ventilatory requirements. ${ }^{2}$ In addition, haemodialysis with a high potassium containing dialysate provides a safe and rapid means for the correction of hypokalaemia and acidosis, and should be considered for all cases of severe barium poisoning, particularly in the presence of respiratory muscle weakness.

3 Phelan DM, Hagley SR, Guerin MD. Is hypokalaemia the cause of paralysis in baum poisoning? BMY 1984;289:882. 4 Gould DB. Barium sulphide poisoning. Some factors contributing to survival. Arch Intern Med 1973;132:891-4. 